\title{
Blow-Up for a Stochastic Viscoelastic Lamé Equation with Logarithmic Nonlinearity
}

\author{
Amina Benramdane, ${ }^{1}$ Nadia Mezouar, ${ }^{2}$ Mohammed Sulaiman Alqawba, ${ }^{3}$ \\ Salah Mahmoud Boulaaras $\mathbb{D}^{3,4}$ and Bahri Belkacem Cherif $\mathbb{D}^{3,5}$ \\ ${ }^{1}$ Mascara University, Faculty of Exact Sciences, Mascara 29000, Algeria \\ ${ }^{2}$ Mascara University, Faculty of Economies Sciences, Mascara 29000, Algeria \\ ${ }^{3}$ Department of Mathematics, College of Sciences and Arts, Qassim University, Ar Rass, Saudi Arabia \\ ${ }^{4}$ Laboratory of Fundamental and Applied Mathematics of Oran (LMFAO), University of Oran 1, Ahmed Benbella, Algeria \\ ${ }^{5}$ Preparatory Institute for Engineering Studies in Sfax, Tunisia
}

Correspondence should be addressed to Bahri Belkacem Cherif; bahi1968@yahoo.com

Received 7 March 2021; Accepted 2 April 2021; Published 19 April 2021

Academic Editor: Chuanjun Chen

Copyright (c) 2021 Amina Benramdane et al. This is an open access article distributed under the Creative Commons Attribution License, which permits unrestricted use, distribution, and reproduction in any medium, provided the original work is properly cited.

In this paper, we consider an initial boundary value problem of stochastic viscoelastic wave equation with nonlinear damping and logarithmic nonlinear source terms. We proved a blow-up result for the solution with decreasing kernel.

\section{Introduction}

In recent years, stochastic partial differential equations in a separable Hilbert space have been studied by many authors, and various results on the existence, uniqueness, stability, blow-up, and other quantitative and qualitative properties of solutions have been established.

In this work, we consider the following problem of stochastic wave equation:

$$
\left\{\begin{array}{l}
\left.u_{t t}-\mu \Delta u-(\lambda+\mu) \nabla(\operatorname{divu})+\int_{0}^{t} h(t-s) \Delta u(s) d s+\left|u_{t}\right|^{q-2} u_{t}=u|u|^{p-2} \ln |u|^{k}+\varepsilon \sigma(x, t) W_{t}(x, t) \text { in } \mathscr{D} \times\right] 0,+\infty[, \\
u(x, t)=0 \text { on } \partial \mathscr{D} \times[0,+\infty[, \\
u(x, 0)=u_{0}(x), u_{t}(x, 0)=u_{1}(x) \text { in } \bar{D},
\end{array}\right.
$$

where $\mathscr{D}$ is a bounded domain in $I R^{n}, n \in \mathrm{I} N^{*}$, with a smooth boundary $\partial \mathscr{D} ; \mu, \lambda$ are the Lamé constants which satisfy $\mu>0$ ,$\lambda+\mu \geq 0 ; h$ is a positive function, $p>q \geq 2$; the constant $k$ is a small nonnegative real number; and $L^{2}(\mathscr{D})$ is the set of square integrable function on $\mathscr{D}$ equipped with the inner product $\langle.,$.$\rangle and its norm \|\cdot\|_{2}$.
$W(x, t)$ is an infinite dimensional Wiener process, $\sigma(x, t)$ is $L^{2}(\mathscr{D})$ valued progressively measurable, and $\varepsilon$ is a positive constant which measures the strength of noise.

It is common to observe a wave motion as a physical phenomenon which is mathematically modeled by a partial differential equation of hyperbolic type. Much has been 
written about such equations regarding their widespread applications to engineering and sciences. However, for more realistic models, the random fluctuation had been taken into consideration which led to introduced stochastic wave equation in 1960's. Several examples of linear stochastic wave propagation and applications can be found in [1]. Mueller [2] was the first who investigate the existence of explosive solutions for some stochastic wave equation. Motivated by Mueller [2], Chow [3] was interested by knowing how does a random perturbation affect the solution behavior for a wave equation with a polynomial nonlinearity. He was concerned with the existence of local and global solutions of the stochastic equation:

$$
\left\{\begin{array}{l}
u_{t t}=\Delta u+f(u)+\sigma(u) W_{t}(x, t) \text { in } x \in \mathbb{R}^{d}, \quad t>0 \\
u(x, 0)=g(x), u_{t}(x, 0)=h(x)
\end{array}\right.
$$

where the initial data $g$ and $h$ are given functions and the nonlinear terms $f(u)$ and $\sigma(u)$ are assumed to be polynomials in $u$. Four years later, he [4] established an energy inequality and the exponential bound for a linear stochastic equation and gave the existence theorem for a unique global solution for the randomly perturbed wave equation:

$\left\{\begin{array}{l}u_{t t}+2 \alpha u_{t}-A(x, \partial x) u(x, t)=f(x, t)+\sigma(x, t) W_{t}(x, t) \text { in } x \in \mathbb{R}^{d}, \quad t>0, \\ u(x, 0)=u_{0}(x), u_{t}(x, 0)=v_{0}(x) .\end{array}\right.$

In 2009, Chow [5] studied the problem of explosive solutions for a class of nonlinear stochastic wave equation in a domain $\mathscr{D} \subset \mathbb{R}^{d}$ for $d \geq 3$,

$$
\left\{\begin{array}{l}
u_{t t}=\left(c^{2} \Delta-\alpha\right) u+f(u)+\sigma(u, x, t) W_{t}(x, t) \text { in } x \in \mathscr{D}, \quad t>0, \\
u(x, 0)=g(x), u_{t}(x, 0)=h(x) .
\end{array}\right.
$$

We can mention some other works such as Cheng et al. [6] who studied the existence of a global solution and blowup solutions for the nonlinear stochastic viscoelastic wave equation with nonlinear damping and source terms:

$$
\left\{\begin{array}{l}
\left.u_{t t}-\Delta u+\int_{0}^{t} h(t-s) \Delta u(s) d s+\left|u_{t}\right|^{q-2} u_{t}=u|u|^{p-2}+\varepsilon \sigma(x, t) W_{t}(x, t) \text { in } \mathscr{D} \times\right] 0,+\infty[ \\
u(x, t)=0 \text { on } \partial \mathscr{D} \times[0,+\infty[ \\
u(x, 0)=u_{0}(x), u_{t}(x, 0)=u_{1}(x) \text { in } \overline{\mathscr{D}}
\end{array}\right.
$$

The authors proved that finite time blow-up with nonnegative probability is explosive or it is explosive in energy sense for $p>q$.
Moreover, Kim et al. [7] considered the stochastic quasilinear viscoelastic wave equation with nonlinear damping and source terms:

$$
\left\{\begin{array}{l}
\left.\left|u_{t}\right| \rho u_{t t}-\Delta u-\Delta u_{t t}+\int_{0}^{t} h(t-s) \Delta u(s) d s+\left|u_{t}\right|{ }^{q-2} u_{t}=u|u|^{p-2}+\varepsilon \sigma(x, t) W_{t}(x, t) \text { in } \mathscr{D} \times\right] 0,+\infty[ \\
u(x, t)=0 \text { on } \partial \mathscr{D} \times[0,+\infty[ \\
u(x, 0)=u_{0}(x), u_{t}(x, 0)=u_{1}(x) \text { in } \overline{\mathscr{D}} .
\end{array}\right.
$$

They showed the existence of a global solution and blowup in finite time.
Recently, Yang et al. [8] treated the following stochastic nonlinear viscoelastic wave equation:

$$
\left\{\begin{array}{l}
\left.\left|u_{t}\right|^{\rho} u_{t t}-\Delta u-\Delta u_{t t}+\int_{0}^{t} h(t-s) \Delta u(s) d s=\sigma(x, t) W_{t}(x, t) \text { in } \mathscr{D} \times\right] 0,+\infty[, \\
u(x, t)=0 \text { on } \partial \mathscr{D} \times[0,+\infty[ \\
u(x, 0)=u_{0}(x), u_{t}(x, 0)=u_{1}(x) \text { in } \overline{\mathscr{D}} .
\end{array}\right.
$$


They established the existence of global solution and asymptotic stability of the solution by using some properties of the convex function.

However, it was noticed that the logarithmic nonlinearity appears naturally in many branches of physics such as nuclear physics, optics, and geophysics (see $[9,10])$. These specific applications in physics and other fields attract a lot of mathematical scientists to work with such problems. In the deterministic case, Al-Gharabli [11] investigated the stability of the solution of a viscoelastic plate equation with a logarithmic nonlinearity source term for the following problem:

$$
\left\{\begin{array}{l}
\left.u_{t t}+\Delta^{2} u+u+\int_{0}^{t} h(t-s) \Delta u^{2}(s) d s=u \ln |u|^{k} \text { in } \mathscr{D} \times\right] 0,+\infty[ \\
\left.u=\frac{\partial u}{\partial v}=0 \text { in } \partial \mathscr{D} \times\right] 0,+\infty[ \\
u(x, 0)=u_{0}(x), u_{t}(x, 0)=u_{1}(x) \text { in } \bar{D},
\end{array}\right.
$$

where $\mathscr{D} \subseteq \mathbb{R}^{2}$ is a bounded domain with a smooth boundary $\partial \mathscr{D}$. The vector $v$ is the unit outer normal to $\partial \mathscr{D}$, and $h$ is the nondecreasing nonnegative function.

Mezouar et al. [12] treated a more general problem where they considered the following nonlinear viscoelastic Kirchhoff equation with a time-varying delay term:

$$
\left\{\begin{array}{l}
\left.\left|u_{t}\right|^{l} u_{t t}-M\left(\|\nabla u\|^{2}\right) \Delta u-\Delta u_{t t}+\int_{0}^{t} h(t-s) \Delta u(s) d s+\mu_{1} g_{1}\left(u_{t}(x, t)\right)+\mu_{2} g_{2}\left(u_{t}(x, t-\tau(t))\right)=k u \ln |u| \text { in } \mathscr{D} \times\right] 0,+\infty[ \\
u(x, t)=0 \text { on } \partial \mathscr{D} \times[0,+\infty[ \\
u(x, 0)=u_{0}(x), u_{t}(x, 0)=u_{1}(x) \text { in } \mathscr{D} \\
\left.u_{t}(x, t-\tau(0))=f_{0}(x, t-\tau(0)) \text { in } \mathscr{D} \times\right] 0, \tau(0)[.
\end{array}\right.
$$

The paper is organized as follows: in Section 2, we introduce some basic definitions, necessary assumptions, and lemmas that are helpful in proving our main result. Section 3 is devoted to show the blow-up of the solution of our problem.

\section{Preliminaries}

Let $(\Omega, \mathscr{F}, P)$ be a complete probability space for which a filtration $\left\{\mathscr{F}_{t}, t \geq 0\right\}$ of increasing sub $\sigma$ - fields $\mathscr{F}_{t}$ is given and $W(x, t)$ be a continuous Wiener random field in this space with a mean zero and the covariance operator $Q$ satisfying

$$
\operatorname{Tr}(Q)=\sum_{i \geq 1} \lambda_{i}<\infty
$$

$$
\begin{aligned}
& W(x, t) \text { is defined by } \\
& W(x, t)=\sum_{j=1}^{\infty} \sqrt{\lambda_{j}} \beta_{j}(t) e_{j}(t), \quad j \in \mathrm{I} N^{*}, t \geq 0,
\end{aligned}
$$

where $\beta_{j}(t)$ is a sequence of real-valued standard Brownian motions mutually independent on the probability space $(\Omega$ $, \mathscr{F}, P), \lambda_{j}$ are the eigenvalues of $Q$, and $e_{j}$ are the corresponding eigenvectors. That is,

$$
Q e_{j}=\lambda_{j} e_{j} .
$$

Note $E($.$) stands for expectation with respect to probabil-$ ity measure $P$. Let $\mathscr{H}$ be the set of $L_{2}^{0}=L^{2}\left(Q^{1 / 2} V, V\right)$-valued processes with the norm

$$
\|\phi(t)\|_{\mathscr{H}}^{2}=E \int_{0}^{t}\|\phi(s)\|_{L_{2}^{0}}^{2} d s=E \int_{0}^{t} \operatorname{Tr}\left(\phi(s) Q \phi^{*}(s)\right) d s<\infty,
$$

where $\phi^{*}(s)$ denotes the adjoint operator of $\phi(s)$ and $V=$ $H_{0}^{1}(\mathscr{D})$ which is equivalent to $H^{1}(\mathscr{D})$. For any process $\phi(s)$ $\in \mathscr{H}$, we can define the stochastic integral with respect to the $Q$-Wiener process as $\int_{0}^{t} \phi(s) d W(s)$ which is a martingale. For more details about the infinite dimension Wiener process and stochastic integral, we refer to Da Prato and Zabczyk (pp. 90-96, [13]).

To state and prove our result, we need some assumptions.

A1. Assume that $h: I R^{+} \longrightarrow I R^{+}$is a $C^{1}$ nonincreasing function satisfying

$$
h(0)>0, \mu-\int_{0}^{\infty} h(s) d s=l>0,
$$

and there exist tow nonnegative constants $\varsigma_{1}$ and $\varsigma_{2}$ such that

$$
-\varsigma_{1} h(t) \leq h^{\prime}(t) \leq-\varsigma_{2} h(t), \quad t \geq 0 .
$$


A2.

$$
\int_{0}^{\infty} h(s) d s<\mu \frac{(p-2) p}{(p-1)^{2}}
$$

A3. $p>q \geq 2$ and

$$
\left\{\begin{array}{l}
2<p \leq \frac{2(n-1)}{n-2}, \quad \text { if } n \geq 3, \\
2<k \leq+\infty, \quad \text { if } n=1,2 .
\end{array}\right.
$$

The following theorem states the existence and uniqueness of a local solution of our problem; the proof can be established by combining the proof given in $[6,12]$.

Theorem 1. Assume that (A1) and (A3) hold. If $\left(u_{0}, u_{1}\right) \in H_{0}^{1}$ $(\mathscr{D}) \times L^{2}(\mathscr{D})$ andE $\int_{0}^{t}\|\sigma(t)\|_{2}^{2} d t<\infty$, then there exists a solution in whichuholds (1) on the interval $[0, T]$ in the sense of distributions over $(0, T) \times \mathscr{D}$ for almost all $w$ a test function such that

$$
\begin{gathered}
\left(u, u_{t}\right) \in L^{2}\left(\Omega ; L^{\infty}\left([0, T] ;\left(H^{2}(\mathscr{D}) \cap H_{0}^{1}(\mathscr{D})\right) \times H_{0}^{1}(\mathscr{D})\right)\right) \\
\cap L^{2}\left(\Omega ; C\left([0, T] ; H_{0}^{1}(\mathscr{D}) \times L^{2}(\mathscr{D})\right)\right) .
\end{gathered}
$$

We define the energy associated to the solution of system (1) by

$$
\begin{aligned}
e(t)= & \frac{1}{2}\left\|u_{t}\right\|_{2}^{2}+\frac{1}{2}\left(\mu-\int_{0}^{t} h(s) d s\right)\|\nabla u\|_{2}^{2} \\
& +\frac{\lambda+\mu}{2}\|\operatorname{div} u\|_{2}^{2}+\frac{1}{2}(h o \nabla u)(t)^{k} d x, \\
& +\frac{k}{p^{2}}\|u\|_{p}^{p}-\frac{1}{p} \int_{\mathscr{D}}|u|^{p} \ln |u|
\end{aligned}
$$

where

$$
(h o v)(t)=\int_{0}^{t} h(t-s)\|v(., t)-v(., s)\|^{2} d s .
$$

We rewrite (1) as an equivalent Itô's system

$$
\left\{\begin{array}{l}
d u=v d t \\
\left.d v=\left[\mu \Delta u+(\lambda+\mu) \nabla(\operatorname{div} u)-\int_{0}^{t} h(t-s) \Delta u(s) d s-|v|^{q-2} v+u|u|^{p-2} \ln |u|^{k}\right] d t+\varepsilon \sigma(x, t) d W_{t}(x, t) \text { in } \mathscr{D} \times\right] 0,+\infty[ \\
u(x, t)=0 \text { on } \partial \mathscr{D} \times[0,+\infty[ \\
u(x, 0)=u_{0}(x), v(x, 0)=u_{1}(x) \text { in } \overline{\mathscr{D}},
\end{array}\right.
$$

which can be written as the integral equation

$$
\left\{\begin{array}{l}
u(t)=u_{0}+\int_{0}^{t} v(s) d s, \\
\left.v(t)=v(0)+\int_{0}^{t}\left[\mu \Delta u+(\lambda+\mu) \nabla(\operatorname{div} u)-\int_{0}^{t} h(s-r) \Delta u(r) d r-|v|^{q-2} v+u|u|^{p-2} \ln |u|^{k}\right] d s+\int_{0}^{t} \varepsilon \sigma(x, s) d W_{s}(x, t) \text { in } \mathscr{D} \times\right] 0,+\infty[, \\
u(x, t)=0 \text { on } \partial \mathscr{D} \times[0,+\infty[, \\
u(x, 0)=u_{0}(x), v(x, 0)=u_{1}(x) \text { in } \bar{D} .
\end{array}\right.
$$

Lemma 2 [14] (Sobolev-Poincaré's inequality). Let $m$ be a or number with

$$
2 \leq m \leq+\infty(n=1,2)
$$

$$
\frac{2 \leq m \leq 2 n}{(n-2)(n \geq 3)}
$$


Then there exists a constant $C_{s}=C_{s}(\mathscr{D}, m)$ such that

$$
\|u\|_{m} \leq C_{s}\|\nabla u\|_{2}, \quad \text { for } u \in H_{0}^{1}(\mathscr{D}) .
$$

Lemma 3 [15]. For $h, \varphi \in C^{1}([0,+\infty[, I R)$, we have

$$
\begin{aligned}
\int_{\mathscr{D}} h * \varphi \varphi_{t} d x= & -\frac{1}{2} h(t)\|\varphi(t)\|_{2}^{2}+\frac{1}{2}\left(h^{\prime} \circ \varphi\right)(t) \\
& -\frac{1}{2} \frac{d}{d t}\left[(h \circ \varphi)(t)-\left(\int_{0}^{t} h(s) d s\right)\|\varphi\|^{2}\right]
\end{aligned}
$$

Lemma 4. Let $(u, v)$ be a solution of the problem (21) with the initial data $\left(u_{0}, v_{0}\right) \in H_{0}^{1}(\mathscr{D}) \times L^{2}(\mathscr{D}), \quad E \int_{0}^{t}\|\sigma(s)\|_{2}^{2} d s<\infty$. Then, the energy functional defined by (19) satisfies

$$
\begin{aligned}
e(t)= & e(0)-\int_{0}^{t}\|v\|_{q}^{q} d s-\frac{1}{2} \int_{0}^{t} h(s)\|\nabla u(s)\|_{2}^{2} d s \\
& +\frac{1}{2} \int_{0}^{t}\left(h^{\prime} o \nabla u\right)(s) d s+\int_{0}^{t}\left\langle v(s), \varepsilon \sigma(x, s) d W_{s}\right\rangle \\
& +\frac{\varepsilon^{2}}{2} \sum_{j=1}^{\infty} \int_{0}^{t} \int_{\mathscr{D}} \lambda_{j} e_{j}^{2}(x) \sigma^{2}(x, s) d x d s .
\end{aligned}
$$

Proof. We can apply the Itô's formula to (21) for each $x \in \mathscr{D}$ after integrating the above equation over $\mathscr{D}$ to get

$$
\begin{aligned}
\|v(t)\|_{2}^{2}= & \|v(0)\|_{2}^{2}+2 \int_{\mathscr{D}} \int_{0}^{t} v(s)[\mu \Delta u+(\lambda+\mu) \nabla(\operatorname{div} u) \\
& \left.-\int_{0}^{s} h(s-\tau) \Delta u(\tau) d \tau-|v|^{q-2} v+u|u|^{p-2} \ln |u|^{k}\right] d s d x \\
& +2 \int_{0}^{t}\left\langle v(s), \varepsilon \sigma(x, s) d W_{s}\right\rangle \\
& +\varepsilon^{2} \sum_{j=1}^{\infty} \int_{0}^{t} \int_{\mathscr{D}} \lambda_{j} e_{j}^{2}(x) \sigma^{2}(x, s) d x d s .
\end{aligned}
$$

By using integration by parts, we get

$$
\begin{aligned}
& \mu \int_{\mathscr{D}} \int_{0}^{t} \Delta u v(s) d x d s=-\mu \int_{\mathscr{D}} \int_{0}^{t} \nabla u \nabla v d s d x \\
&=-\frac{\mu}{2}\left(\|\nabla u(t)\|_{2}^{2}-\|\nabla u(0)\|_{2}^{2}\right), \\
& \int_{\mathscr{D}} \int_{0}^{t}(\lambda+\mu) \nabla(\operatorname{div} u(s)) v(s) d s d x \\
&=-(\lambda+\mu) \int_{\mathscr{D}} \int_{0}^{t} \operatorname{div} u(s) \operatorname{div} v(s) d s d x \\
&=-\frac{\lambda+\mu}{2}\left[\|\operatorname{div} u(t)\|_{2}^{2}-\|\operatorname{div} u(0)\|_{2}^{2}\right] .
\end{aligned}
$$

By applying Lemma 3, we have

$$
\begin{aligned}
& \int_{0}^{t} \int_{\mathscr{D}} \int_{0}^{s} h(s-\tau) \Delta u(\tau) v(s) d \tau d x d s \\
& =-\int_{0}^{t} \int_{\mathscr{D}} \int_{0}^{s} h(s-\tau) \nabla u(\tau) \nabla v(s) d \tau d x d s \\
& =\int_{0}^{t}\left(\frac{1}{2} h(s)\|\nabla u(s)\|_{2}^{2}-\frac{1}{2}\left(h^{\prime} o \nabla u\right)(s)\right. \\
& \left.\left.\quad+\frac{1}{2} \frac{d}{d s}\left[(h o \nabla u)(s)-\int_{0}^{s} h(\tau) d \tau\right)\|\nabla u(s)\|_{2}^{2}\right]\right) d s .
\end{aligned}
$$

We have

$$
\begin{aligned}
& \int_{\mathscr{D}} \int_{0}^{t} u|u|^{p-2} \ln |u|^{k} u_{s} d s d x \\
& \quad=\int_{0}^{t} \int_{\mathscr{D}} \frac{1}{\mathrm{p}} \frac{d}{d s}\left(|u(s)|^{p}\right) \ln |u|^{k} d x d s \\
& =\int_{0}^{t}\left\{\int _ { \mathscr { D } } \left\{\frac{1}{p} \frac{d}{d s}\left(|u(s)|^{p} \ln |u|^{k}\right)\right.\right. \\
& \left.\left.\quad-\frac{1}{p}|u(s)|^{p} \frac{d}{d s}\left(\ln |u|^{k}\right)\right\} d x\right\} d s \\
& =\int_{\mathscr{D}}\left(\frac{1}{p}\left(|u|^{p} \ln |u|^{k}\right)\right) d x-\frac{k}{p^{2}}\|u\|_{p}^{p} .
\end{aligned}
$$

By replacing (29)-(32) in (28) and multiplying equation (28) by $1 / 2$, we arrive at (27).

\section{Blow-Up}

We prove our main result for $p>q$; we purpose

$$
\begin{aligned}
E \int_{0}^{\infty} \int_{\mathscr{D}} \sigma^{2}(x, t) d x d t<\infty \\
G(t)=\frac{\varepsilon^{2}}{2} \sum_{j=1}^{\infty} E \int_{0}^{t} \int_{\mathscr{D}} \lambda_{j} e_{j}^{2}(x) \sigma^{2}(x, s) d x d s \\
G(\infty)=\frac{\varepsilon^{2}}{2} \sum_{j=1}^{\infty} E \int_{0}^{\infty} \int_{\mathscr{D}} \lambda_{j} e_{j}^{2}(x) \sigma^{2}(x, s) d x d s \\
\leq \frac{\varepsilon^{2}}{2} \operatorname{Tr}(Q) c_{0}^{2} E \int_{0}^{\infty} \int_{\mathscr{D}} \sigma^{2}(x, s) d x d s:=E_{1}<\infty
\end{aligned}
$$

where

$$
\operatorname{Tr}(Q)=\sum_{j=1}^{\infty} \lambda_{j}<\text { oand } c_{0}=\sup _{j \geq 1}\left\|e_{j}\right\|_{\infty}<\infty
$$


Lemma 5. Let $(u, v)$ be a solution of system (21) with initial data $\left(u_{0}, v_{0}\right) \in H_{0}^{1}(\mathscr{D}) \times L^{2}(\mathscr{D})$. Then, we have

$$
\begin{aligned}
\frac{d}{d t} E e(t)= & -E\|v(t)\|_{q}^{q}-\frac{1}{2} h(t) E\|\nabla u(t)\|_{2}^{2}+\frac{1}{2} E\left(h^{\prime} o \nabla u\right)(t) \\
& +\frac{\varepsilon^{2}}{2} \sum_{j=1}^{\infty} E \int_{D} \lambda_{j} e_{j}^{2}(x) \sigma^{2}(x, t) d x
\end{aligned}
$$

$$
\begin{aligned}
E\langle u(t), v(t)\rangle= & E\left\langle u_{0}, u_{1}\right\rangle-\mu \int_{0}^{t} E\|\nabla u(s)\|_{2}^{2} d s \\
& -(\lambda+\mu) \int_{0}^{t} E\|\operatorname{div} u(s)\|_{2}^{2} d s \\
& +E \int_{0}^{t} \int_{0}^{s} h(s-r)\langle\nabla u(r), \nabla u(s)\rangle d r d s \\
& -E \int_{0}^{t}\left\langle u(s),|v(s)|^{q-2} v(s)\right\rangle d s \\
& +E \int_{0}^{t}\left\langle u(s), u(s)|u(s)|^{p-2} \ln |u(s)|^{k}\right\rangle d s \\
& +E \int_{0}^{t}\|v(s)\|_{2}^{2} d s .
\end{aligned}
$$

Proof. Using the Itô's formula and by following the same way as our discussions in Lemma 4 with taking the expectations, we obtain (37).

We multiply the second equation in (22) by $u$ and integrate the result over $\mathscr{D}$, and we take expectation; we obtain (38).

We set $H(t)=G(t)-E e(t)$. As $h$ is a positive decreasing function so

$$
\begin{aligned}
H^{\prime}(t)= & G^{\prime}(t)-\frac{d}{d t} E e(t)=E\|v\|_{q}^{q}+\frac{1}{2} h(t) E\|\nabla u(t)\|_{2}^{2} \\
& -\frac{1}{2} E\left(h^{\prime} o \nabla u\right)(t) \geq E\|v\|_{q}^{q} .
\end{aligned}
$$

Consequently,

$$
H^{\prime}(t) \geq 0
$$

Lemma 6. Let $(u, v)$ be a solution of system (21). Assume that (A1) holds. Then, there exists a positive constant $C$ such that

$$
\begin{aligned}
E\|u(t)\|_{p+1}^{s} \leq & C\left(G(t)-H(t)-\frac{1}{2} E\|v\|_{2}^{2}\right. \\
& +\frac{1}{p} E \int_{\mathscr{D}}|u|^{p} \ln |u|^{k} d x-\frac{1}{2} E(h o \nabla u)(t) \\
& \left.-\frac{\lambda+\mu}{2} E\|\operatorname{div} u\|_{2}^{2}+E\|u\|_{p+1}^{p+1}\right),
\end{aligned}
$$

where $2 \leq s \leq p+1$.
Proof.

$$
\begin{aligned}
G(t) & -H(t)-\frac{1}{2} E\|v\|_{2}^{2}+\frac{1}{p} E \int_{\mathscr{D}}|u|^{p} \ln |u|^{k} d x \\
& -\frac{1}{2} E(h o \nabla u)(t)-\frac{\lambda+\mu}{2} E\|\operatorname{div} u\|_{2}^{2}+E\|u\|_{p+1}^{p+1} \\
= & E e(t)-\frac{1}{2} E\|v\|_{2}^{2}+\frac{1}{\mathrm{p}} E \int_{\mathscr{D}}|u|^{p} \ln |u|^{k} d x \\
& -\frac{1}{2} E(h o \nabla u)(t)+E\|u\|_{p+1}^{p+1}-\frac{\lambda+\mu}{2}\|\operatorname{div} u\|_{2}^{2} \\
= & \frac{1}{2} E\left\|u_{t}\right\|_{2}^{2}+\frac{1}{2} E\left(\mu-\int_{0}^{t} h(s) d s\right)\|\nabla u\|_{2}^{2} \\
& +\frac{\lambda+\mu}{2} E\|\operatorname{div} u\|_{2}^{2}+\frac{1}{2} E(h o \nabla u)(t)+\frac{k}{p^{2}} E\|u\|_{p}^{p} \\
& -\frac{1}{p} E \int_{\mathscr{D}}|u|^{p} \ln |u|^{k} d x-\frac{1}{2} E\|v\|_{2}^{2} \\
& +\frac{1}{p} E \int_{\mathscr{D}}|u|^{p} \ln |u|^{k} d x-\frac{1}{2} E(h o \nabla u)(t) \\
& -\frac{\lambda+\mu}{2} E\|\operatorname{div} u\|_{2}^{2}+E\|u\|_{p+1}^{p+1} \\
= & \frac{1}{2}\left(\mu-\int_{0}^{t} h(s) d s\right) E\|\nabla u\|_{2}^{2}+\frac{k}{p^{2}} E\|u\|_{p}^{p}+E\left\|^{2} u\right\|_{p+1}^{p+1} \\
& l E u\left\|_{2}^{2}+E\right\| u \|_{p+1}^{p+1} \cdot
\end{aligned}
$$

The last inequality is getting from (A1).

Case 7. If $\|u\|_{p+1} \leq 1$, then $\|u\|_{p+1}^{s} \leq\|u\|_{p+1}^{2}$.

By applying Lemma 2, we obtain $\|u\|_{p+1}^{s} \leq c\|\nabla u\|_{2}^{2}$, then $\frac{1}{2} l E\|\nabla u\|_{2}^{2}+\frac{k}{p^{2}} E\|u\|_{p+1}^{p+1} \geq \frac{1}{2} l E\|u\|_{p+1}^{s}+\frac{k}{p^{2}} E\|u\|_{p+1}^{p+1} \geq E\|u\|_{p+1}^{s}$.

Case 8. If $\|u\|_{p+1} \geq 1$, then $\|u\|_{p+1}^{p+1} \geq\|u\|_{p+1}^{s}$.

Hence,

$$
\frac{1}{2} l E\|\nabla u\|_{2}^{2}+E\|u\|_{p+1}^{p+1} \geq \frac{1}{2} l E\|\nabla u\|_{2}^{2}+E\|u\|_{p+1}^{s} \geq E\|u\|_{p+1}^{s} .
$$

Consequently, we obtain (41).

We are ready to state and prove our main result for $p>q$. For this purpose, we define

$$
L(t):=H^{1-\alpha}(t)+\delta E\langle u, v\rangle
$$

where

$$
0<\alpha<\min \left\{\frac{p-1}{2(p+1)}, \frac{p+1-q}{(p+1) q}\right\}
$$

and $\delta$ is a very small constant determined later. 
Theorem 9. Assume (A1) and (A2) hold. Let $(u, v)$ be a solution of system (21) with initial data $\left(u_{0}, v_{0}\right) \in H_{0}^{1}(\mathscr{D}) \times L^{2}(\mathscr{D})$ satisfying

$$
\operatorname{Ee}(0) \leq-(1+\beta) E_{1},
$$

where $\beta$ is a nonnegative constant and $E_{1}$ is given in (35). If $p>q$, then there exists a positive time $T_{0} \in[0, T]$ such that

$$
\lim _{t \longrightarrow T_{0}^{-}} E(e(t))=+\infty
$$

where

$$
\begin{gathered}
T_{0}=\frac{1-\alpha}{\alpha K L^{\alpha / 1-\alpha}(0)}, \\
L(0)=H^{1-\alpha}(0)+\delta E\left\langle u_{0}, u_{1}\right\rangle>0,
\end{gathered}
$$

and $K$ is given later.

Proof. Let

$$
L(t)=H^{1-\alpha}(t)+\delta E\langle u, v\rangle .
$$

A direct differentiation of $L(t)$ gives

$$
\begin{aligned}
L^{\prime}(t)= & (1-\alpha) H^{-\alpha}(t) H^{\prime}(t)+\delta\left[-\mu E\|\nabla u(t)\|_{2}^{2}\right. \\
& -(\lambda+\mu) E\|\operatorname{divu}\|_{2}^{2}+E \int_{0}^{t} h(t-r)\langle\nabla u(r), \nabla u(t)\rangle d r \\
& -E\left\langle u(t),|v(t)|^{q-2} v(t)\right\rangle \\
& \left.+E\left\langle u(t), u(t)|u(t)|^{p-2} \ln |u(t)|^{k}\right\rangle+E\|v(t)\|_{2}^{2}\right] \\
= & (1-\alpha) H^{-\alpha}(t) H^{\prime}(t)+\delta\left[-\mu E\|\nabla u(t)\|_{2}^{2}\right. \\
& -(\lambda+\mu) E\|\operatorname{div} u\|_{2}^{2}+E \int_{0}^{t} h(t-r)\langle\nabla u(r), \nabla u(t)\rangle d r \\
& -E\left\langle u(t),|v(t)|^{q-2} v(t)\right\rangle+E\left\langle u(t), u(t)|u(t)|^{p-2} \ln |u(t)|^{k}\right\rangle \\
& \left.+E\|v(t)\|_{2}^{2}\right]+\delta p[H(t)-G(t)+E e(t)] .
\end{aligned}
$$

Recalling (39) and (19), (51) leads to

$$
\begin{aligned}
L^{\prime}(t) \geq & (1-\alpha) H^{-\alpha}(t) E\|v\|_{q}^{q}+\delta p(H(t)-G(t)) \\
& +\delta\left(\frac{\mu p}{2}-\mu\right) E\|\nabla u(t)\|_{2}^{2}+\delta\left(\frac{p}{2}+1\right) E\|v\|_{2}^{2} \\
& -\delta E\left\langle u(t),|v(t)|^{q-2} v(t)\right\rangle-\frac{\delta p}{2} E \int_{0}^{t} h(s) d s\|\nabla u\|_{2}^{2} \\
& +\delta E \int_{0}^{t} h(t-r)\langle\nabla u(r), \nabla u(t)\rangle d r \\
& +(\lambda+\mu) \delta\left(\frac{\mathrm{p}}{2}-1\right) E\|\operatorname{div} u\|_{2}^{2}+\frac{\delta k}{p} E\|u\|_{p}^{p} \\
& +\frac{\delta p}{2} E(h o \nabla u)(t) .
\end{aligned}
$$

By using Young's and Hölder's inequalities, we get

$$
\begin{aligned}
E \int_{0}^{t} h(t-r)\langle\nabla u(r), \nabla u(t)\rangle d r \\
=E \int_{0}^{t} h(t-r)\langle\nabla u(r)-\nabla u(t), \nabla u(t)\rangle d r+E \int_{0}^{t} h(s) d s\|\nabla u(t)\|_{2}^{2} \\
\geq-\frac{p}{2} E(h o \nabla u)(t)-\frac{1}{2 p} E \int_{0}^{t} h(s) d s\|\nabla u(t)\|_{2}^{2} \\
\quad+E \int_{0}^{t} h(s) d s\|\nabla u(t)\|_{2}^{2} .
\end{aligned}
$$

Hence,

$$
\begin{aligned}
L^{\prime}(t) \geq & (1-\alpha) H^{-\alpha}(t) E\|v\|_{q}^{q}+\delta p(H(t)-G(t)) \\
& +\delta\left(\frac{\mu p}{2}-\mu\right) E\|\nabla u(t)\|_{2}^{2}+\delta\left(\frac{p}{2}+1\right) E\|v\|_{2}^{2} \\
& -\delta E\left\langle u(t),|v(t)|^{q-2} v(t)\right\rangle \\
& +\delta\left(1-\frac{1}{2 p}-\frac{p}{2}\right) E \int_{0}^{t} h(s) d s\|\nabla u(t)\|_{2}^{2} \\
& -\frac{\delta p}{2} E(h o \nabla u)(t)+\frac{\delta p}{2} E(h o \nabla u)(t) \\
& +(\lambda+\mu) \delta\left(\frac{p}{2}-1\right) E\|\operatorname{div} u\|_{2}^{2}+\frac{\delta k}{p} E\|u\|_{p}^{p} \\
\geq & (1-\alpha) H^{-\alpha}(t) E\|v\|_{q}^{q}+\delta p(H(t)-G(t)) \\
& +\delta\left(\frac{\mu p}{2}-\mu\right) E\|\nabla u(t)\|_{2}^{2}+\delta\left(\frac{p}{2}+1\right) E\|v\|_{2}^{2} \\
& -\delta E\left\langle u(t),|v(t)|^{q-2} v(t)\right\rangle \\
& +\delta\left(1-\frac{1}{2 p}-\frac{p}{2}\right) E \int_{0}^{t} h(s) d s\|\nabla u(t)\|_{2}^{2} \\
& +(\lambda+\mu) \delta\left(\frac{p}{2}-1\right) E\|\operatorname{div} u\|_{2}^{2}+\frac{\delta k}{p} E\|u\|_{p}^{p} .
\end{aligned}
$$

As $q<p+1$, then $E\|u(t)\|_{q}^{q} \leq c E\|u(t)\|_{p+1}^{p+1}$ so by using Young's and Hölder's inequality; we obtain

$$
\begin{aligned}
E\left\langle u(t),|v(t)|^{q-2} v(t)\right\rangle \leq\left(E\|v(\mathrm{t})\|_{q}^{q}\right)^{q-1 / q}\left(E\|u(t)\|_{q}^{q}\right)^{1 / q} \\
\leq c\left(E\|v(t)\|_{q}^{q}\right)^{q-1 / q}\left(E\|u(t)\|_{p+1}^{p+1}\right)^{1 / p+1} \\
\left.\left.\leq c\left(E\|v(t)\|_{q}^{q}\right)^{q-1 / q}\left(E\|u(t)\|_{p+1}^{p+1}\right)\right)^{(1 / p+1)-(1 / q)}\left(E\|u(t)\|_{p+1}^{p+1}\right)\right)^{(1 / q)} \\
\leq c\left(\frac{q-1}{q} \xi\left(E\|v(t)\|_{q}^{q}\right)+\frac{\xi^{1-q}}{q}\left(E\|u(t)\|_{p+1}^{p+1}\right)\right) \\
\left.\quad \times\left(E\|u(t)\|_{p+1}^{p+1}\right)\right)^{(1 / p+1)-(1 / q)}
\end{aligned}
$$

where $\xi$ and $c$ are constants.

We consider the following partition of $\mathscr{D}$ :

$$
\mathscr{D}_{1}=\{x \in \mathscr{D}:|u|>1\}, \mathscr{D}_{2}=\{x \in \mathscr{D}:|u| \leq 1\} .
$$


We have

$$
\begin{aligned}
E \int_{\mathscr{D}}|u|^{p} \ln |u|^{k} d x & =E \int_{\mathscr{D}_{1}}|u|^{p} \ln |u|^{k} d x+E \int_{\mathscr{D}_{2}}|u|^{p} \ln |u|^{k} d x \\
& \leq E \int_{\mathscr{D}_{1}}|u|^{p} \ln |u|^{k} d x \\
& \leq E \int_{\mathscr{D}_{1}} k|u|^{p+1} d x \\
& \leq k E\|u\|_{p+1}^{p+1} .
\end{aligned}
$$

By (40), (47), and $-E e(0)=H(0)$, we have

$$
\begin{gathered}
(1+\beta) G(t)<(1+\beta) E_{1} \leq H(0) \leq H(t) \\
\leq G(t)+\frac{1}{p} E \int_{\mathscr{D}}|u|^{p} \ln |u|^{k} d x .
\end{gathered}
$$

Therefore,

$$
G(\mathrm{t}) \leq \frac{1}{1+\beta} H(t)
$$

From (57), (58), and (59), we get

$k E\|u(t)\|_{p+1}^{p+1} \geq E \int_{\mathscr{D}_{1}} k|u|^{p+1} d x \geq p(H(t)-G(t)) \geq p \frac{\beta}{1+\beta} H(t)$.

As $H$ is increasing positive nonnegative function and by recalling (46), we get

$$
\begin{aligned}
(E & \left.\|u(t)\|_{p+1}^{p+1}\right)^{(1 / p+1)-(1 / q)} \\
& \leq\left(p \frac{\beta}{k(1+\beta)}\right)^{(1 / p+1)-(1 / q)} H^{(1 / p+1)-(1 / q)}(t) \\
& \leq\left(p \frac{\beta}{k(1+\beta)}\right)^{(1 / p+1)-(1 / q)} H^{-\alpha}(t) \\
& \leq\left(p \frac{\beta}{k(1+\beta)}\right)^{(1 / p+1)-(1 / q)} H^{-\alpha}(0) .
\end{aligned}
$$

Taking into account (61) in (55), we find

$$
\begin{aligned}
E\left\langle u(t),|v(t)|^{q-2} v(t)\right\rangle & \\
\leq & \left(c\left(\frac{p \beta}{k(1+\beta)}\right)^{(1 / p+1)-(1 / q)}\right) \frac{\mathrm{q}-1}{q} \xi\left(E\|v(t)\|_{q}^{q}\right) H^{-\alpha}(t) \\
& +\left(c\left(\frac{p \beta}{k(1+\beta)}\right)^{(1 / p+1)-(1 / q)}\right) \frac{\xi^{1-q}}{q}\left(E\|u(t)\|_{p+1}^{p+1}\right) H^{-\alpha}(0) .
\end{aligned}
$$

Substituting (62) into (54), we get

$$
\begin{aligned}
L^{\prime}(t) \geq & (1-\alpha) H^{-\alpha}(t) E\|v\|_{q}^{q}+\delta p(H(t)-G(t)) \\
& +\delta \mu\left(\frac{p}{2}-1\right) E\|\nabla u(t)\|_{2}^{2}+\delta\left(\frac{p}{2}+1\right) E\|v\|_{2}^{2} \\
& +\delta\left(1-\frac{1}{2 p}-\frac{p}{2}\right) E \int_{0}^{t} h(s) d s\|\nabla u(t)\|_{2}^{2} \\
& +(\lambda+\mu) \delta\left(\frac{p}{2}-1\right) E\|\operatorname{div} u\|_{2}^{2} \\
& -\delta \frac{a_{1}(q-1)}{q} \xi\left(E\|v\|_{q}^{q}\right) H^{-\alpha}(t) \\
& -\delta \frac{a_{1}}{q} \xi^{1-q}\left(E\|u\|_{p+1}^{p+1}\right) H^{-\alpha}(0)+\frac{\delta k}{p} E\|u\|_{p}^{p},
\end{aligned}
$$

where $a_{1}=c(p \beta /(k(1+\beta)))^{(1 / p+1)-(1 / q)}$.

Using Lemma 6, we arrive at

$$
\begin{aligned}
L^{\prime}(t) \geq & \left(1-\alpha-\delta \frac{a_{1}(q-1)}{q} \xi\right) H^{-\alpha}(t) E\|v\|_{q}^{q}+\delta p(H(t) \\
& -G(t))+\delta \mu\left(\frac{p}{2}-1\right) E\|\nabla u(t)\|_{2}^{2}+\delta\left(\frac{p}{2}+1\right) E\|v\|_{2}^{2} \\
& +\delta\left(1-\frac{1}{2 p}-\frac{p}{2}\right) E \int_{0}^{t} h(s) d s\|\nabla u(t)\|_{2}^{2} \\
& +(\lambda+\mu) \delta\left(\frac{p}{2}-1\right)\|\operatorname{div} u\|_{2}^{2}-\delta \frac{a_{1}}{q} \xi^{1-q} H^{-\alpha}(0) C \\
& \cdot\left(G(t)-H(t)-\frac{1}{2} E\|v\|_{2}^{2}+\frac{1}{p} E \int_{\mathscr{D}}^{|u|^{p} \ln |u|^{k} d x}\right. \\
& \left.+E\|u\|_{p+1}^{p+1}-\frac{1}{2} E(h o \nabla u)(t)-\frac{\lambda+\mu}{2} E\|\operatorname{div} u\|_{2}^{2}\right) \\
& +\frac{\delta k}{p} E\|u\|_{p}^{p} .
\end{aligned}
$$

Once $\xi$ is fixed, we pick $\delta$ small enough so that

$$
1-\alpha-\delta \frac{a_{1}(q-1)}{q} \xi \geq 0
$$

It implies that

$$
\begin{aligned}
L^{\prime}(t) \geq & \delta\left(p+a_{2} \xi^{1-q}\right)(H(t)-G(t)) \\
& +\delta\left(\frac{p}{2}+1+a_{2} \frac{1}{2} \xi^{1-q}\right) E\|v\|_{2}^{2} \\
& -\delta a_{2} \xi^{1-q} \frac{1}{p} E \int_{\mathscr{D}}|u|^{p} \ln |u|^{k} d x+\delta(\lambda+\mu) \\
& \cdot\left(\xi^{1-q} \frac{a_{2}}{2}+\left(\frac{p}{2}-1\right)\right) E\|\operatorname{div} u\|_{2}^{2} \\
& +\delta a_{2} \xi^{1-q} \frac{1}{2} E(h o \nabla u)(t)+\delta a_{3} E\|\nabla u(t)\|_{2}^{2} \\
& +\frac{\delta k}{p} E\|u\|_{p}^{p}-\delta a_{2} \xi^{1-q} E\|u\|_{p+1}^{p+1} .
\end{aligned}
$$


where $a_{2}=C\left(a_{1} / q\right) H^{-\alpha}(0)$ and $a_{3}=\mu((p / 2)-1)+(1-(1 /$ $2 p)-(p / 2)) \int_{0}^{\infty} h(s) d s$ which is positive from (A2).

From (A1), (19), and Lemma 2, we have

$$
\begin{aligned}
H(t)-G(t) \geq & -\frac{1}{2} E\|v\|_{2}^{2}-\left(\frac{\mu}{2}+2 C_{s}\right) E\|\nabla u\|_{2}^{2} \\
& -\frac{\lambda+\mu}{2} E\|\operatorname{div} u\|_{2}^{2}-\frac{1}{2} E(h o \nabla u)(t) \\
& -\frac{k}{p^{2}} E\|u\|_{p}^{p}+\frac{1}{p} E \int_{\mathscr{D}}|u|^{p} \ln |u|^{k} d x+E\|\mathrm{u}\|_{p+1}^{p+1} .
\end{aligned}
$$

Now we add and subtract $\delta a_{4}(H(t)-G(t))$ in (66), and using (67), we find

$$
\begin{aligned}
L^{\prime}(t) \geq & \delta\left(p-a_{4}+a_{2} \xi^{1-q}\right)(H(t)-G(t)) \\
& +\delta\left(\frac{p}{2}+1-\frac{a_{4}}{2}+a_{2} \frac{1}{2} \xi^{1-q}\right) E\|v\|_{2}^{2} \\
& +\delta(\lambda+\mu)\left(\xi^{1-q} \frac{a_{2}}{2}+\left(\frac{p}{2}-1\right)-\frac{a_{4}}{2}\right) E\|\operatorname{div} u\|_{2}^{2} \\
& +\delta\left(a_{2} \xi^{1-q} \frac{1}{2}-a_{4}\right) E(h o \nabla u)(t) \\
& +\delta\left(a_{3}-a_{4}\left(\frac{\mu+4 C_{s}}{2}\right)\right) E\|\nabla u(t)\|_{2}^{2} \\
& +\frac{\delta k}{p}\left(1-\frac{a_{4}}{p}\right) E\|u\|_{p}^{p}+\frac{\delta}{p}\left(a_{4}-a_{2} \xi^{1-q}\right) E \int_{\mathscr{D}}|u|^{p} \ln |u|^{k} d x \\
& +\delta\left(a_{4}-a_{2} \xi^{1-q}\right) E\|u\|_{p+1}^{p+1},
\end{aligned}
$$

where $a_{4}=\min \left\{a_{2} \xi^{1-q},\left(2 a_{3} /\left(\mu+4 C_{s}\right)\right)\right\}>0$.

Using (60), we obtain

$$
\begin{aligned}
L^{\prime}(\mathrm{t}) \geq & \delta p \frac{\beta}{1+\beta} H(t)+\delta\left(\frac{p}{2}+1-\frac{a_{4}}{2}+a_{2} \frac{1}{2} \xi^{1-q}\right) E\|v\|_{2}^{2} \\
& +\delta(\lambda+\mu)\left(\xi^{1-q} \frac{a_{2}}{2}+\left(\frac{p}{2}-1\right)-\frac{a_{4}}{2}\right) E\|\operatorname{div} u\|_{2}^{2} \\
& +\frac{\delta}{2}\left(a_{2} \xi^{1-q}-a_{4}\right) E(h o \nabla u)(t) \\
& +\delta\left(a_{3}-a_{4}\left(\frac{\mu+4 C_{s}}{2}\right)\right) E\|\nabla u(t)\|_{2}^{2} \\
& +\frac{\delta k}{p}\left(1-\frac{a_{4}}{p}\right) E\|u\|_{p}^{p}+\delta\left(a_{4}-a_{2} \xi^{1-q}\right) E\|u\|_{p+1}^{p+1} \\
\geq & \gamma\left(H(t)+E\|v\|_{2}^{2}+E\|\operatorname{div} u\|_{2}^{2}+E(h o \nabla u)(t)\right. \\
& \left.+E\|\nabla u(t)\|_{2}^{2}+E\|u\|_{p}^{p}+E\|u\|_{p+1}^{p+1}\right) \geq 0,
\end{aligned}
$$

where $\gamma>0$ is the minimum of the coefficients of $H(t), E\|v\|_{2}^{2}$, $E\|\operatorname{div} u\|_{2}^{2}, E(h o \nabla u)(t), E\|\nabla u(t)\|_{2}^{2}$, and $E\|u\|_{p}^{p}$ in (69).

Consequently,

$$
L(t) \geq L(0)>0, \quad \forall t>0 .
$$

Next, we have

$$
\begin{aligned}
(L(t))^{1 / 1-\alpha} & =\left(H^{1-\alpha}(t)+\delta E\langle u, v\rangle\right)^{1 / 1-\alpha} \\
& \leq 2^{1 / 1-\alpha}\left(H(t)+\delta^{1 / 1-\alpha}\left|E \int_{\mathscr{D}} u v d x\right|^{1 / 1-\alpha}\right) .
\end{aligned}
$$
obtain

$$
\begin{aligned}
& \left|E \int_{D} u v d x\right|^{1 / 1-\alpha} \leq\left(c\left(E\|u\|_{p+1}^{2}\right)^{1 / 2}\left(E\|v\|_{2}^{2}\right)^{1 / 2}\right)^{1 / 1-\alpha} \\
& \quad \leq c\left(E\|u\|_{p+1}^{2}\right)^{1 /(2(1-\alpha))}\left(E\|v\|_{2}^{2}\right)^{1 /(2(1-\alpha))} \\
& \quad \leq c\left[\frac{\left(E\|u\|_{p+1}^{2}\right)^{\eta /(2(1-\alpha))}}{\eta}+\frac{\left(E\|v\|_{2}^{2}\right)^{\zeta /(2(1-\alpha))}}{\zeta}\right],
\end{aligned}
$$

with $(1 / \eta)+(1 / \zeta)=1$.

We choose $=2(1-\alpha), \eta=(2(1-\alpha)) / 1-2 \alpha$, and we use (46), so (72) becomes

$$
\begin{aligned}
& \left|E \int_{\mathscr{D}} u v d x\right|^{1 / 1-\alpha} \leq c\left[(1-2 \alpha) E\|u\|_{p+1}^{2 / 1-2 \alpha}+E\|v\|_{2}^{2}\right] \\
& \quad \leq c\left[E\|u\|_{p+1}^{2 / 1-2 \alpha}+E\|v\|_{2}^{2}\right] .
\end{aligned}
$$

By applying Lemma 6 with $s=2 / 1-2 \alpha$ and recalling (19), we obtain

$$
\begin{aligned}
&\left|E \int_{\mathscr{D}} u v d x\right|^{1 / 1-\alpha} \leq c\left[G(t)-H(t)-\frac{1}{2} E\|v\|_{2}^{2}\right. \\
&+\frac{1}{p} E \int_{\mathscr{D}}|u|^{p} \ln |u|^{k} d x-\frac{1}{2} E(h o \nabla u)(t) \\
&\left.\quad-\frac{\lambda+\mu}{2} E\|\operatorname{div} u\|_{2}^{2}+E\|u\|_{p+1}^{p+1}+E\|v\|_{2}^{2}\right] \\
& \leq c\left[\frac{1}{2} E\|v\|_{2}^{2}+\frac{1}{2}\left(\mu-\int_{0}^{t} h(s) d s\right) E\|\nabla u\|_{2}^{2}\right. \\
&+\frac{\lambda+\mu}{2} E\|\operatorname{div} u\|_{2}^{2}+\frac{k}{p^{2}} E\|u\|_{p}^{p}+\frac{1}{2} E(h o \nabla u)(t) \\
&-\frac{1}{p} E \int_{\mathscr{D}}|u|^{p} \ln |u|^{k} d x-\frac{1}{2} E\|v\|_{2}^{2}+\frac{1}{p} E \int_{\mathscr{D}}|u|^{p} \ln |u|^{k} d x \\
&\left.-\frac{1}{2} E(h o \nabla u)(t)-\frac{\lambda+\mu}{2} E\|\operatorname{div} u\|_{2}^{2}+E\|v\|_{2}^{2}+E\|u\|_{p+1}^{p+1}\right] \\
& \leq c\left[E\|v\|_{2}^{2}+\frac{1}{2} \mu E\|\nabla u\|_{2}^{2}+\frac{k}{p^{2}} E\|u\|_{p}^{p}+\frac{\lambda+\mu}{2} E\|\operatorname{div} u\|_{2}^{2}\right. \\
&\left.+\frac{1}{2} E(h o \nabla u)(t)+E\|u\|_{p+1}^{p+1}\right] .
\end{aligned}
$$


Hence,

$$
\begin{aligned}
&(L(t))^{1 / 1-\alpha} \leq 2^{1 / 1-\alpha}\left(H(t)+\delta^{1 / 1-\alpha} c\left[E\|v\|_{2}^{2}+\frac{1}{2} \mu E\|\nabla u\|_{2}^{2}\right.\right. \\
&\left.\left.+\frac{\lambda+\mu}{2} E\|\operatorname{div} u\|_{2}^{2}+\frac{k}{p^{2}} E\|u\|_{p}^{p}+\frac{1}{2} E(h o \nabla u)(t)+E\|u\|_{p+1}^{p+1}\right]\right) \\
& \leq \tilde{C}\left[H(t)+E\|v\|_{2}^{2}+E\|\nabla u\|_{2}^{2}+E\|\operatorname{div} u\|_{2}^{2}+E\|u\|_{p}^{p}\right. \\
&\left.+E(h o \nabla u)(t)+E\|u\|_{p+1}^{p+1}\right],
\end{aligned}
$$

where $\quad \tilde{C}=2^{1 / 1-\alpha} \max \left\{1, c \delta^{1 / 1-\alpha}, c \delta^{1 / 1-\alpha}((\lambda+\mu) / 2), c \delta^{1 / 1-\alpha}\right.$ $\left.\left(k / p^{2}\right)\right\}$.

According to (69) and (75), we have

$$
(L(t))^{1 / 1-\alpha} \leq \frac{\tilde{C}}{\gamma} L^{\prime}(t) \leq \tilde{K} L^{\prime}(t) .
$$

In a direct integration of (76), we get

$$
(L(t))^{1 / 1-\alpha} \geq \frac{1}{(L(0))^{1 / 1-\alpha}-(\tilde{K} \alpha t /(1-\alpha))} .
$$

Therefore, $L(t)$ blows up in time $T \leq T_{0}=(1-\alpha) /$ $\left(\alpha K L^{\alpha / 1-\alpha}(0)\right)$, and the proof is completed.

\section{Data Availability}

No data were used to support the study.

\section{Conflicts of Interest}

This work does not have any conflicts of interest.

\section{References}

[1] P. L. Chow, W. Kohler, and G. Papanicolaou, Multiple Scattering and Waves in Random Media, North-Holland Publishing Company, North-Holland, Amsterdam, 1981.

[2] C. Mueller, "Long time existence for the wave equation with a noise term," The Annals of Probability, vol. 25, no. 1, pp. 133151, 1997.

[3] P. L. Chow, "Stochastic wave equations with polynomial nonlinearity," The Annals of Applied Probability, vol. 12, no. 1, pp. 361-381, 2002.

[4] P. L. Chow, "Asymptotics of solutions to semilinear stochastic wave equations," The Annals of Applied Probability, vol. 16, no. 2, pp. 757-789, 2006.

[5] P. L. Chow, "Nonlinear stochastic wave equations: blow-up of second moments in L2-norm," Annals of Applied Probability, vol. 19, no. 6, pp. 2039-2046, 2009.

[6] S. Cheng, Y. Guo, and Y. Tang, "Stochastic viscoelastic wave equations with nonlinear damping and source terms," Journal of Applied Mathematics, vol. 2014, 15 pages, 2014.

[7] S. Kim, J. Y. Park, and Y. H. Kang, "Stochastic quasilinear viscoelastic wave equation with nonlinear damping and source terms," Boundary Value Problems, vol. 2018, no. 1, Article ID 14, 2018.
[8] H. Yang, S. Fang, F. Liang, and M. Li, "A general stability result for second order stochastic quasilinear evolution equations with memory," Boundary Value Problems, vol. 2020, no. 1, Article ID 62, 2020.

[9] K. Enqvist and J. McDonald, "Q-balls and baryogenesis in the MSSM," Physics Letters B, vol. 425, no. 3-4, pp. 309-321, 1998.

[10] P. Górka, "Logarithmic Klein-Gordon equation," Acta Physica Polonica B, vol. 40, no. 1, pp. 59-66, 2009.

[11] M. M. Al-Gharabli, "New general decay results for a viscoelastic plate equation with a logarithmic nonlinearity," BBoundary Value Problems, vol. 2019, no. 1, article 194, 2019.

[12] N. Mezouar, S. Boulaaras, and A. Allahem, "Global existence of solutions for the viscoelastic Kirchhoff equation with logarithmic source terms," Complexity, vol. 2020, Article ID 7105387, 25 pages, 2020.

[13] G. Da Prato and J. Zabczyk, Stochastic Equations in Infinite Dimensions, Cambridge University Press, 1992.

[14] R. A. Adams, "Sobolev espaces, academic press," Pure and Applied Mathematics, vol. 65, 1978.

[15] J. Y. Park and J. R. Kang, "Global existence and uniform decay for a nonlinear viscoelastic equation with damping," Acta Applicandae Mathematicae, vol. 110, no. 3, pp. 1393-1406, 2010. 